\title{
SDSS J103913.70+533029.7: A Super Star Cluster in the Outskirts of a Galaxy Merger
}

\section{Citation}

Knapp, Gillian R., Christy A. Tremonti, Constance M. Rockosi, David J. Schlegel, Brian Yanny, Timothy C. Beers, Carlos Allende Prieto, et al. 2006. “SDSS J103913.70+533029.7: A Super Star Cluster in the Outskirts of a Galaxy Merger." The Astronomical Journal 131 (2) (February): 859865. doi:10.1086/499304.

\section{Published Version}

doi:10.1086/499304

\section{Permanent link}

http://nrs.harvard.edu/urn-3:HUL.InstRepos:33461893

\section{Terms of Use}

This article was downloaded from Harvard University's DASH repository, and is made available under the terms and conditions applicable to Other Posted Material, as set forth at http:// nrs.harvard.edu/urn-3:HUL.InstRepos:dash.current.terms-of-use\#LAA

\section{Share Your Story}

The Harvard community has made this article openly available.

Please share how this access benefits you. Submit a story.

\section{Accessibility}




\title{
SDSS J103913.70+533029.7: A SUPER STAR CLUSTER IN THE OUTSKIRTS OF A GALAXY MERGER
}

\author{
Gillian R. Knapp, ${ }^{1}$ Christy A. Tremonti, ${ }^{2}$ Constance M. Rockosi, ${ }^{3}$ David J. Schlegel, ${ }^{4}$ Brian Yanny, ${ }^{5}$ \\ Timothy C. Beers, ${ }^{6}$ Carlos Allende Prieto, ${ }^{7}$ Ron Wilhelm,${ }^{8}$ Robert H. Lupton, ${ }^{1}$ James E. Gunn, ${ }^{1}$ \\ Martin Niederste-Ostholt, ${ }^{1}$ Donald P. Schneider, ${ }^{9}$ Kevin Covey, ${ }^{10}$ Anil Seth, ${ }^{10}$ Željko Ivezić, ${ }^{10}$ \\ Daniel J. Eisenstein, ${ }^{2}$ Joe Helmboldt, ${ }^{11}$ Douglas P. Finkbeiner, ${ }^{1}$ Nikhil Padmanabhan, ${ }^{12}$ \\ Scot J. Kleinman, ${ }^{13}$ Dan Long, ${ }^{13}$ Stephanie A. Snedden, ${ }^{13}$ Atsuko Nitta, ${ }^{13}$ \\ Michael Harvanek, ${ }^{13}$ Jurek Krzesinski, ${ }^{13,14}$ Howard J. Brewington, ${ }^{13}$ \\ John C. Barentine, ${ }^{13}$ Peter R. Newman, ${ }^{13}$ Eric H. Nielsen, Jr., \\ Masataka Fukugita, ${ }^{15}$ and J. Brinkmann ${ }^{13}$ \\ Received 2005 August 11; accepted 2005 October 21
}

\begin{abstract}
We describe the serendipitous discovery in the spectroscopic data of the Sloan Digital Sky Survey of a starlike object, SDSS J103913.70+533029.7, at a heliocentric radial velocity of $+1012 \mathrm{~km} \mathrm{~s}^{-1}$. Its proximity in position and velocity to the spiral galaxy NGC 3310 suggests an association with the galaxy. At this distance, SDSS J103913.70+533029.7 has the luminosity of a super star cluster and a projected distance of $17 \mathrm{kpc}$ from NGC 3310. Its spectroscopic and photometric properties imply a mass of $>10^{6} M_{\odot}$ and an age close to that of the tidal shells seen around NGC 3310, suggesting that it formed in the event that formed the shells.
\end{abstract}

Key words: galaxies: formation — galaxies: individual (NGC 3310) — galaxies: starburst — galaxies: star clusters

\section{INTRODUCTION}

Super star clusters (van den Bergh 1971; Arp \& Sandage 1985; Schweizer 1986; Lançon \& Boily 2000; Lamers et al. 2004; Gallagher \& Smith 2004) are compact (diameters less than $20 \mathrm{pc})$, high-luminosity $\left(M_{V}<-10\right)$ clusters whose properties resemble those of globular clusters except that they are young, often much less than $1 \mathrm{Gyr}$ in age. As such, they are important for our understanding of the formation of globular cluster systems and, by extension, of the galaxies of which they are members.

Large numbers of these systems have been discovered and characterized in the last 15 years or so, especially from high angular resolution imaging with the Hubble Space Telescope (HST) and spectroscopic observations with new, very large groundbased optical telescopes. While super star clusters have not yet been seen in the Milky Way or in M31, they have been discov-

\footnotetext{
${ }^{1}$ Department of Astrophysical Sciences, Princeton University, Princeton, NJ 08544.

2 Department of Astronomy and Steward Observatory, University of Arizona, 933 North Cherry Avenue, Tucson, AZ 85721-0065.

3 Lick Observatory, University of California, Santa Cruz, CA 95064.

4 Lawrence Berkeley Laboratory, 1 Cyclotron Road, Berkeley, CA 94720.

5 Fermi National Accelerator Laboratory, P.O. Box 500, Batavia, IL 60510.

6 Department of Physics and Astronomy and Joint Institute for Nuclear Astrophysics (JINA), Michigan State University, East Lansing, MI 48824.

${ }^{7}$ McDonald Observatory and Department of Astronomy, University of Texas, Austin, TX 78712.

8 Department of Physics, Texas Tech University, Lubbock, TX 79409.

9 Department of Astronomy and Astrophysics, Pennsylvania State University, University Park, PA 16802.

${ }^{10}$ Department of Astronomy, University of Washington, Box 351580, Seattle, WA 98195.

11 Department of Astronomy, New Mexico State University, Box 30001, Department 4500, Las Cruces, NM 88003-8001.

12 Department of Physics, Joseph Henry Laboratories, Princeton University, Princeton, NJ 08544.

13 Apache Point Observatory, P.O. Box 59, Sunspot, NM 88349.

14 Mount Suhora Observatory, Cracow Pedagogical Observatory, ul. Podchorazych 2, 30-084 Cracow, Poland.

${ }^{15}$ Institute for Cosmic Ray Research, University of Tokyo, 5-1-5 Kashiwa, Kashiwa City, Chiba 277-8582, Japan.
}

ered in large numbers in several environments: in starbursting gas-rich dwarf irregular galaxies (Melnick et al. 1985; O'Connell et al. 1994; Ho \& Filippenko 1996a, 1996b; Gelatt et al. 2001; Billett et al. 2002; Vanzi 2003); in more massive starburst galaxies (Holtzmann et al. 1992; Whitmore et al. 1993; Meurer et al. 1995; Whitmore \& Schweizer 1995; Zepf et al. 1999; Tremonti et al. 2001; Lipscy \& Plavchan 2004; Turner \& Beck 2004; McCrady et al. 2005; Melo et al. 2005), many of which show signs of recent mergers or interactions; and in the compact group Stephan's Quintet (Gallagher et al. 2001). In some of these galaxies, such as M82, NGC 4038/4039, and NGC 1275, super star clusters incorporate a significant fraction of the starburst (Lipscy \& Plavchan 2004; McCrady et al. 2005) and are preferentially found in the inner regions of the galaxy (Whitmore \& Schweizer 1995; Meurer et al. 1995). Their masses are measured to be up to $10^{6} M_{\odot}$ (McCrady et al. 2005), and their metallicities range from $[\mathrm{Fe} / \mathrm{H}]=-1.5$ in dwarf irregular galaxies to several times solar in the large galaxy mergers.

NGC 3310 is a small spiral galaxy $\left(M_{B}=-19.6 ; D=14 \mathrm{Mpc}\right.$, adopting the Hubble distance found using $H_{0}=72 \mathrm{~km} \mathrm{~s}^{-1}$ $\mathrm{Mpc}^{-1}$ ) that has a disturbed morphology in both its optical light and H I distribution (Kregel \& Sancisi 2001; Mulder \& van Driel 1996; de Grijs et al. 2003a, 2003b; Wehner \& Gallagher 2005) and whose inner regions underwent a burst of star formation about $30 \mathrm{Myr}$ ago, with the formation of several hundred super star clusters (de Grijs et al. 2003a). The present paper describes the serendipitous discovery of SDSS J103913.70+533029.7 (hereafter SDSS 1039+53), an unresolved $\left(<1^{\prime \prime}\right)$ object with a radial velocity of about $1000 \mathrm{~km} \mathrm{~s}^{-1}$. Several lines of evidence suggest that it is associated with NGC 3310 . If so, its luminosity, colors, and spectrum point to its being a compact, high-luminosity super star cluster at a projected distance of $17 \mathrm{kpc}$ from NGC 3310. By contrast, all previously known super star clusters lie in the inner kiloparsec or two of their parent galaxy.

The relevant details of the Sloan Digital Sky Survey (SDSS) observations are summarized in $\S 2$. Section 3 describes the properties and nature of SDSS $1039+53$. We discuss the possibility that this object is a high-velocity Galactic star but favor 
TABLE 1

SDSS J103913.70+533029.7

\begin{tabular}{|c|c|}
\hline Parameter & Value \\
\hline 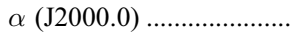 & 103913.70 \\
\hline$\delta(\mathrm{J} 2000.0) \ldots \ldots \ldots \ldots \ldots \ldots \ldots \ldots \ldots \ldots \ldots$ & +533029.7 \\
\hline$v_{\text {helio }}\left(\mathrm{km} \mathrm{s}^{-1}\right) \ldots \ldots \ldots \ldots \ldots \ldots$ & $1012 \pm 19$ \\
\hline$v_{\mathrm{GSR}}\left(\mathrm{km} \mathrm{s}^{-1}\right) \ldots \ldots \ldots \ldots \ldots$ & $1067 \pm 19$ \\
\hline u ........................ & $20.90 \pm 0.09$ \\
\hline ................. & $19.57 \pm 0.02$ \\
\hline$r$ & $19.36 \pm 0.02$ \\
\hline$i$ & $19.19 \pm 0.02$ \\
\hline$z \ldots \ldots \ldots \ldots \ldots \ldots \ldots \ldots \ldots \ldots \ldots \ldots \ldots \ldots \ldots \ldots \ldots \ldots \ldots \ldots \ldots$ & $19.03 \pm 0.04$ \\
\hline
\end{tabular}

Notes. - The magnitudes are corrected for interstellar extinction using the data of Schlegel et al. (1998). Units of right ascension are hours, minutes, and seconds, and units of declination are degrees, arcminutes, and arcseconds.

the evidence associating it with NGC 3310. Under this assumption, its properties are shown to be consistent with its being a super star cluster of an age similar to that of the recent merger/ interaction involving NGC 3310. The SDSS imaging also confirms the presence of stellar shells around NGC 3310 (Wehner $\&$ Gallagher 2005) and shows the disturbed morphology of the galaxy. The discussion and conclusions are given in $\S 4$.

\section{THE SLOAN DIGITAL SKY SURVEY}

The SDSS is a five-band photometric survey of about $10,000 \mathrm{deg}^{2}$ of the northern sky to a depth of about $22.5(r$ magnitude, point source) and a concurrent redshift survey of up to 1 million galaxies and 100,000 quasars selected from the imaging survey (York et al. 2000). The SDSS camera (Gunn et al. 1998), mounted on a dedicated $2.5 \mathrm{~m}$ telescope (Gunn et al. 2005) at Apache Point Observatory, New Mexico, acquires imaging data in five bands, $u, g, r, i$, and $z$, centered at approximate effective wavelengths of $3551,4686,6166,7480$, and $8932 \AA$, respectively (Fukugita et al. 1996). The imaging data are automatically reduced through a series of software pipelines (Lupton et al. 2001, 2002; Pier et al. 2003; Ivezić et al. 2004). The instrumental fluxes are calibrated via a network of primary and secondary stellar flux standards to $\mathrm{AB}_{\nu}$ magnitudes (Oke \& Gunn 1983; Fukugita et al. 1996; Hogg et al. 2001; Smith et al. 2002; Tucker et al. 2005), and the absolute positions are calibrated using standard astrometric catalogs (Pier et al. 2003).

Targets for spectroscopy are selected from the imaging data on the basis of their photometric properties. As well as the primary SDSS targets (galaxies and quasars), stars in many different locations of color-magnitude space are selected to provide backup targets in regions of low galaxy density and serve as spectrophotometric standards. The target objects are mapped (Blanton et al. 2003) onto $3^{\circ}$ diameter aluminum fiber plug plates that feed the spectrographs. The pair of dual fiber-fed spectrographs (Uomoto et al. 1999) can observe 640 spectra at one time with a wavelength coverage of $3800-9200 \AA$ and a resolution of $1800-2100$. The spectra are extracted from CCD images, calibrated, and corrected for sky emission and absorption. The resulting calibrated spectra are fit to a series of templates of galaxies, quasars, and stars to derive the spectral classification, redshift, and redshift error of each object (D. Schlegel 2006, in preparation) ${ }^{16}$ Stellar templates are taken directly from SDSS spectra and calibrated with respect to spectral type and radial velocity using the
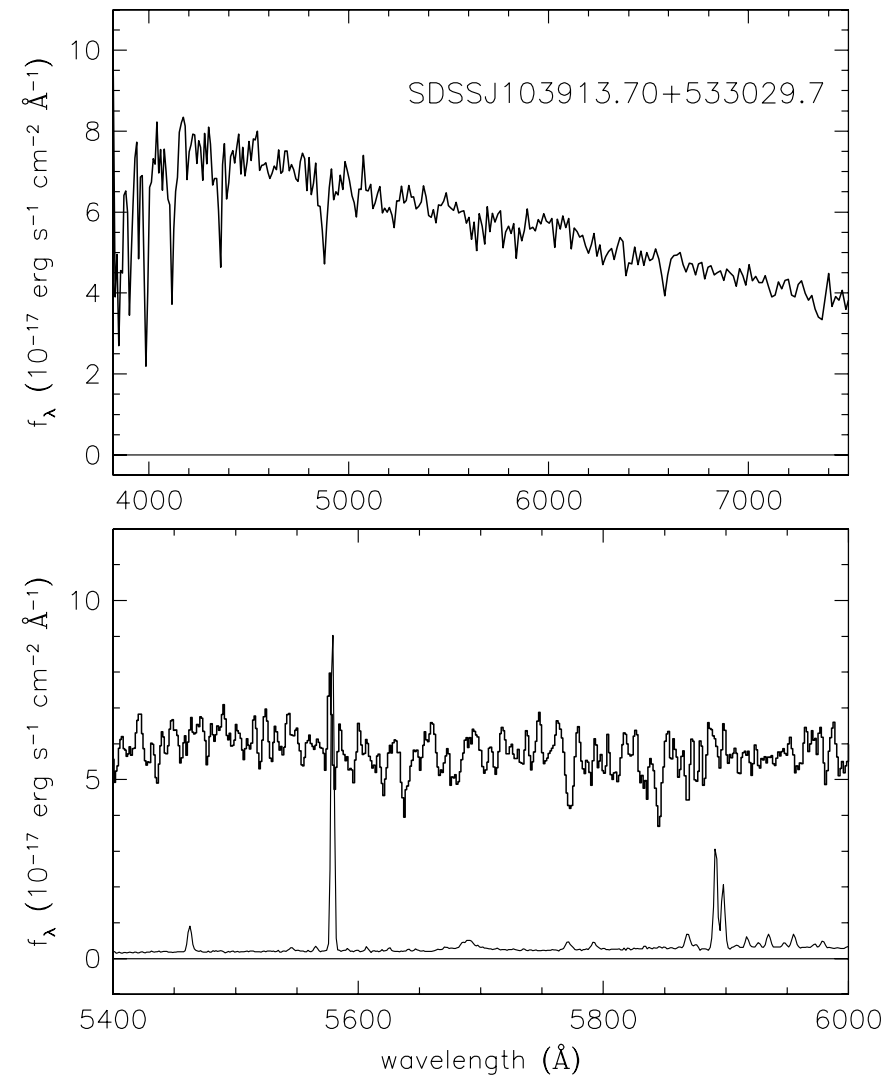

FIG. 1.-SDSS spectrum of SDSS $1039+53$; the spectral resolution is $\approx 2000$. Bottom: Expanded region of the spectrum near the atmospheric $\mathrm{Hg}$ I $\lambda 5460$, [O I] $\lambda 5577$, and $\mathrm{Na}$ I $\mathrm{D}$ lines. The thin line shows the sky spectrum, where these lines are observed at the rest wavelengths. The spectrum is from plate 1010, fiber 335, MJD 52,649 and is available at the SDSS Data Release 3 Web site (see footnote 17).

ELODIE stellar library (Prugniel \& Soubiran 2001; Moultaka et al. 2004). The SDSS data are described in the data release papers by Abazajian et al. $(2003,2004,2005)$ and Adelman-McCarthy et al. (2006) and documented at Web sites listed therein. ${ }^{17}$

We examined the spectroscopic data in the SDSS archives as of 2005 January 12, selecting objects spectroscopically classified as stars. The sample was further selected by magnitude $(g<20)$ and by color, $0.8<u-g<1.5, g-r<0.5$, roughly the colors of halo turnoff stars ( $\mathrm{F}$ subdwarfs) and horizontalbranch stars (cf. Yanny et al. 2000; Newberg et al. 2002; Sirko et al. 2004a). During the selection, magnitudes were not corrected for interstellar extinction. SDSS has obtained spectra of many tens of thousands of stars with these properties; in all, 40,746 spectra were found in the database that satisfied these selection criteria. The heliocentric radial velocities of the stars were corrected to the Galactic standard of rest, assuming a solar velocity of $16.6 \mathrm{~km} \mathrm{~s}^{-1}$ toward $\alpha(\mathrm{J} 2000.0)=17^{\mathrm{h}} 49^{\mathrm{m}} 58^{\mathrm{s}} 7$, $\delta(\mathrm{J} 2000.0)=+28^{\circ} 07^{\prime} 04^{\prime \prime}$ and a motion of the local standard of rest of $220 \mathrm{~km} \mathrm{~s}^{-1}$ toward $\alpha(\mathrm{J} 2000.0)=21^{\mathrm{h}} 12^{\mathrm{m}} 01^{\mathrm{s}} .1$, $\delta(\mathrm{J} 2000.0)=+48^{\circ} 19^{\prime} 47^{\prime \prime}$. The distribution of velocities for this large sample is Gaussian, with a standard deviation of $102 \mathrm{~km} \mathrm{~s}^{-1}$ (Sirko et al. 2004b); however, one object, SDSS 1039+53, has a galactocentric velocity well outside this range, $+1067 \pm 19 \mathrm{~km} \mathrm{~s}^{-1}$, greatly in excess of the expected escape velocity from the Galaxy. Various properties of SDSS $1039+53$ are summarized in Table 1. Its SDSS spectrum is shown in Figure 1, and its colors,

\footnotetext{
${ }^{16} \mathrm{See}$ http://spectro.princeton.edu.
}

\footnotetext{
${ }^{17}$ See also http://www.sdss.org.
} 

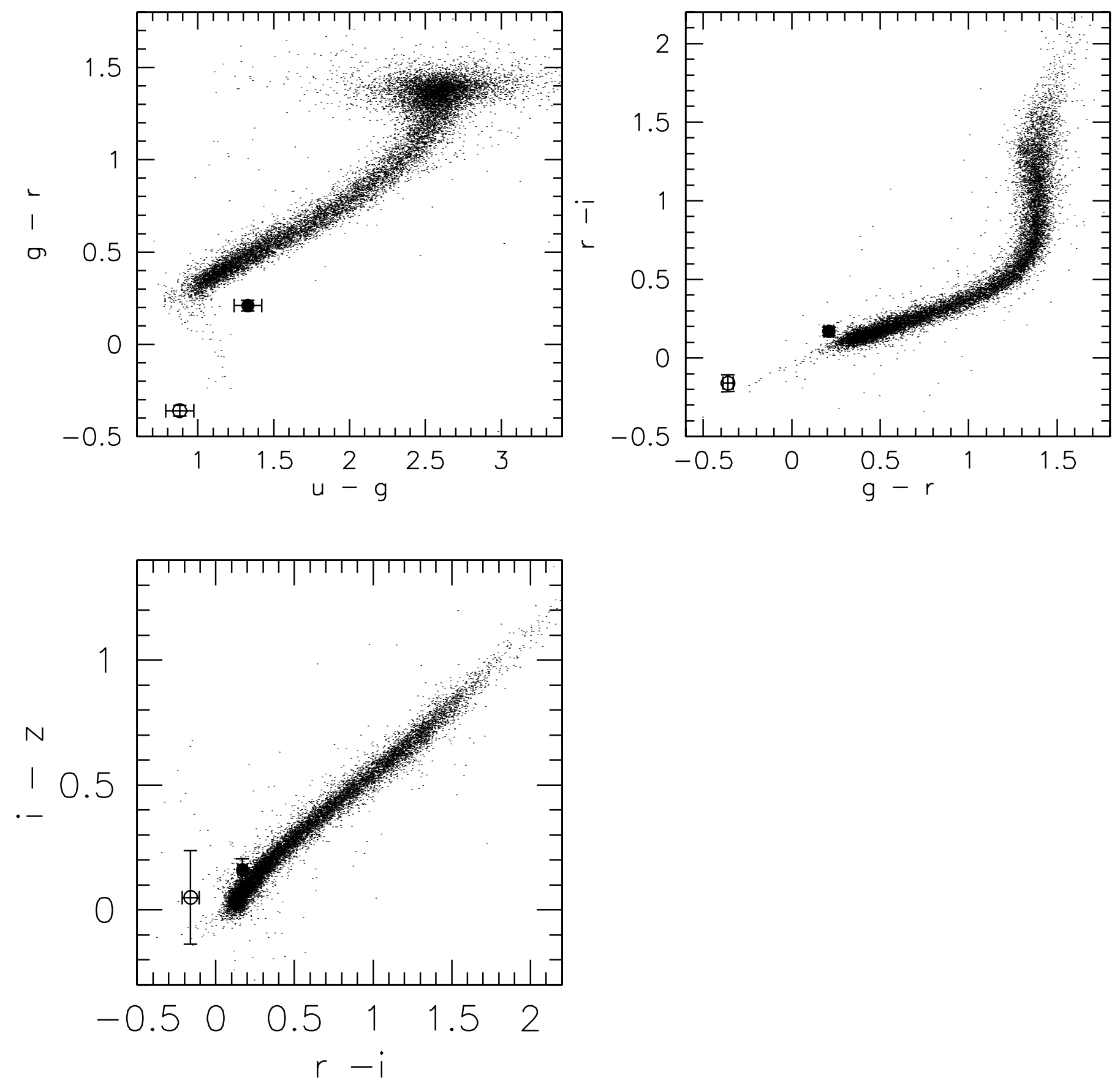

FIG. 2.-SDSS color-color plots. The dots show the colors of a sample of $\sim 15,000$ anonymous stars measured by SDSS (see Finlator et al. 2000). The large filled circles show the colors of the super star cluster SDSS 1039+53, and the open circles show the colors of SDSS J090745.0+024507, the hypervelocity Galactic star described by Brown et al. (2005) (see text for discussion). Note that the colors of SDSS 1039+53 are similar, but not identical, to those of F stars.

corrected for Galactic extinction using the values given by Schlegel et al. (1998), are compared with those of a sample of stars observed by SDSS from Finlator et al. (2000) in Figure 2.

\section{SDSS 1039+53: GALACTIC OR EXTRAGALACTIC?}

SDSS $1039+53$ was observed in two imaging runs. In both observations, the object is classified as a point source in all five bands, except for the $u$-band image taken in poorer seeing (run 2735). In the run with better seeing (run 2821) the object is unresolved at the point-spread function diameter, $00^{\prime \prime} 9$, in the $r$ and $i$ bands. Its colors (Fig. 2) are close to but not identical to those of halo/thick disk F/G stars. The object has no detectable proper motion; USNO-B + SDSS gives $\mu_{\alpha}=1 \pm 3.9, \mu_{\delta}=$

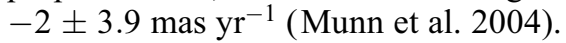

There is only one spectrum of SDSS 1039+53 in the SDSS data archives, that shown in Figure 1, and it is similar to that of a middle F star. Thus, at first glance SDSS 1039+53 appears to be a star with a velocity well outside the velocity range of Galactic stars, and it is the only such object so far discovered in the SDSS spectroscopic database. Given the extraordinary value of the radial velocity, we performed a number of tests to investigate the reality of this measurement. First, the spectrum was analyzed using two different codes and template sets. Cross-correlation analysis (SubbaRao et al. 2002) gives a heliocentric velocity of 
$1028 \pm 23 \mathrm{~km} \mathrm{~s}^{-1}$, while a direct $\chi^{2}$ minimization fit (D. J. Schlegel 2004, unpublished) gives $1012 \pm 19 \mathrm{~km} \mathrm{~s}^{-1}$. The use of different templates, with types from late $\mathrm{B}$ to $\mathrm{F}$, produces a scatter of about $10 \mathrm{~km} \mathrm{~s}^{-1}$, and fitting a polynomial to the centers of the Balmer lines gives $1009 \pm 9 \mathrm{~km} \mathrm{~s}^{-1}$. Second, the spectrum is actually the composite of two spectra, measured by the red and blue spectrographs. Both yield the same radial velocity. Third, there are five stars on plate 1010 that are also observed on other plates, and the measured radial velocities agree to within the errors. In total, there are 30 stars in the selected color and magnitude range observed in plate 1010 in addition to SDSS 1039+53, and the measured velocities span the range -140 to $+213 \mathrm{~km} \mathrm{~s}^{-1}$. Fourth, the sky spectrum was examined. Figure 1 shows the sky spectrum in the region 5400-6000 $\AA$, where very strong nightsky emission from the $\mathrm{Na} \mathrm{D}$ lines and the $\left[\mathrm{O}_{\mathrm{I}}\right] \lambda 5577$ airglow lie. Were there an error in the wavelength calibration of the spectrum of SDSS 1039+53, the night-sky subtraction would show very large residuals in this wavelength region, when in fact there is no discernible residual at Na D and only a small residual at $5577 \AA$.

A final and definitive test was supplied by a spectrum obtained with the Marcario Low-Resolution Spectrograph (Hill et al. 1998) on the Hobby-Eberly Telescope on 2005 February 14. This spectrum, which covered the $\mathrm{H} \alpha$ line at a resolution of approximately 1000 , yields a heliocentric radial velocity of $1033 \pm 70 \mathrm{~km} \mathrm{~s}^{-1}$, consistent with the results from the SDSS data.

Therefore, SDSS $1039+53$ could be a Galactic high-velocity star, like that recently discovered by Brown et al. (2005). Several methods were used to derive the stellar parameters under this assumption. Fitting the flux-calibrated spectrum to synthetic spectra based on Kurucz $(1979,1993)$ model atmospheres using the methods of Allende Prieto et al. (2006) gives $T_{\text {eff }}=6237 \pm$ $247 \mathrm{~K}, \log g=2.00 \pm 0.26$, and $[\mathrm{Fe} / \mathrm{H}]=-0.37 \pm 0.17$ and badly overestimates the strength of the Ca II K line. The techniques described by Wilhelm et al. (1999) yield $T_{\text {eff }}=6456 \mathrm{~K}$, $\log g=2.27$, and $[\mathrm{Fe} / \mathrm{H}]=-2.44$, with the abundance derived primarily from the $\mathrm{Ca}$ II $\mathrm{K}$ line. Other metal lines give a much higher value, $[\mathrm{Fe} / \mathrm{H}]=-0.71$. The spectrum and colors are consistent with classification of SDSS $1039+53$ as a horizontalbranch star at $60 \mathrm{kpc}$. However, the line widths for $\mathrm{H} \epsilon$ and $\mathrm{H} \delta$ are much larger than that of $\mathrm{H} \gamma$. Finally, the abundance obtained using the Beers et al. (1999) calibration of the Ca II K line and the colors is $[\mathrm{Fe} / \mathrm{H}]=-1.88$. Taken as a whole, these results suggest that the spectrum of this object is composite, which might be expected if it is not a single star but the integrated light from a cluster of stars.

If, on the other hand, SDSS $1039+53$ is extragalactic, as suggested by its radial velocity, its very compact light distribution (with an inferred diameter of $\leq 40 \mathrm{pc}$ ) suggests two possible interpretations: it may be a compact dwarf galaxy, like those formed by tidal disruption in clusters (Drinkwater et al. 2003; Haşegan et al. 2005), or it may be a star cluster, like those seen in starburst galaxies. SDSS $1039+53$ does not lie in a cluster of galaxies but is about 4.' 1 from the Sbc galaxy NGC 3310 , which has a very similar radial velocity, $V_{\text {helio }}=993 \mathrm{~km} \mathrm{~s}^{-1}$. Furthermore, NGC 3310 itself is a galaxy that has recently undergone a merger with one or more gas-rich systems; it has extensive $\mathrm{H}$ i tidal tails and is surrounded by a low surface brightness shell system (Kregel \& Sancisi 2001; Wehner \& Gallagher 2005). Thus, the more likely interpretation is that SDSS $1039+53$ is a compact star cluster associated with NGC 3310.

\section{DISCUSSION AND CONCLUSIONS}

NGC 3310 is at a distance of about $14 \mathrm{Mpc}$ and is a "minor merger," with extended H I tidal tails (Kregel \& Sancisi 2001), stellar shells surrounding the main disk (Wehner \& Gallagher 2005), and a large-scale starburst in its inner regions (de Grijs et al. 2003a) that is considerably younger than the inferred age of the merger (see below). The SDSS image of this galaxy and its surroundings is shown in Figure 3. Tidal shells can be seen, as well as the actively star-forming spiral arms, which have a distorted and chaotic morphology. The image also shows SDSS $1039+53$, at a distance of 4.'14 from the center of NGC 3310 . The coincidence in velocity and sky position strongly suggests that these two unusual objects are associated. If so, the absolute magnitude of SDSS $1039+53$ is $M_{r}=-11.3\left(M_{V} \sim-11.2\right.$, using the conversions given by Fukugita et al. 1996). This luminosity is much higher than those of the Galactic globular clusters (cf. Peterson 1993 and Djorgovski 1993) but is similar to those of super star clusters.

What is remarkable about SDSS $1039+53$ is its distance from the galaxy nucleus (4.'14 [17 kpc]). Meurer et al. (1995) note that super star clusters are typically found "at the very heart of starbursts." In their ultraviolet imaging survey, over $90 \%$ of super star clusters were found where the local surface brightness was within $1.5 \mathrm{mag} \operatorname{arcsec}^{-2}$ of its peak value. SDSS $1039+53$, in contrast, is well outside the optical extent of NGC 3310, as seen in Figure 3, and considerably beyond the de Vaucouleurs radius $\left(R_{25}=1.55\right.$; de Vaucouleurs et al. 1991). The cluster's projected distance is equivalent to $\sim 20$ disk scale lengths $\left[r_{0}(I)=\right.$ 12 ".4 $=0.84 \mathrm{kpc}$; Sánchez-Portal et al. 2004]. This would be untypical, but not unheard of, for a globular cluster; roughly 95\% of the Milky Way's globular clusters are found within $\sim 10 r_{0}\left(r_{0}=2.8 \mathrm{kpc}\right.$; Ojha 2001), with the most distant globular cluster lying at $42 r_{0}(117 \mathrm{kpc}$; Harris 1996).

To determine an age for the star cluster, we fit Bruzual \& Charlot (2003) simple stellar population (SSP) models to both the optical spectrum and the SDSS ugriz colors (both the spectrum and colors were corrected for Galactic foreground reddening and extinction using the maps of Schlegel et al. [1998]). Because the cluster lies so far outside the optical and $\mathrm{H}$ i disk of NGC 3310, we assumed no intrinsic reddening. We adopted a metallicity of $Z=0.008$ (60\% solar), since this is in good accord with the nebular abundances in $\mathrm{H}$ II regions outside the nucleus (Pastoriza et al. 1993) and with metallicities derived for star clusters from HST multiband photometry (de Grijs et al. 2003a). We compared the data to a grid of 110 models with ages ranging from 10 Myr to 1 Gyr. The $\chi^{2}$ evolution of the models, along with the best fits, is shown in Figure 4. The optical spectrum is fit over the full wavelength range available, 3800-9200 $\AA$. The best-fit spectrum has an age of 570 Myr. The photometry provides additional information, because the SDSS spectra do not cover the $u$ and $z$ bands; the photometry alone fits best to an SSP model of age $720 \mathrm{Myr}$. These two independent fits provide some measure for the systematic uncertainties in the SDSS photometry and spectroscopy and give a cluster age of $700 \pm 150 \mathrm{Myr}$. The model cluster mass is $1.4 \times 10^{6} M_{\odot}$, assuming a Chabrier (2003) Galactic initial mass function (a Salpeter IMF extending down to $0.1 M_{\odot}$ would increase the mass estimate by a factor of 1.4 ).

The derived age and mass of SDSS $1039+53$ place it squarely in the regime of super star clusters (age $\lesssim 1$ Gyr, mass $=$ $10^{5}-10^{8} M_{\odot}$; O'Connell 2004). However, when compared to the inner disk cluster population in NGC 3310, SDSS 1039+53 stands out as being both relatively old and massive. De Grijs et al. (2003a, 2003b) characterize the cluster population in the inner $33^{\prime \prime}$ of NGC 3310 using multiband HST photometry. Out of 174 clusters, they find only a few percent with $M \sim 10^{6} M_{\odot}$ and roughly $10 \%$ with ages $>100$ Myr. Only one cluster satisfies both criteria. The mean age of the cluster population is around 


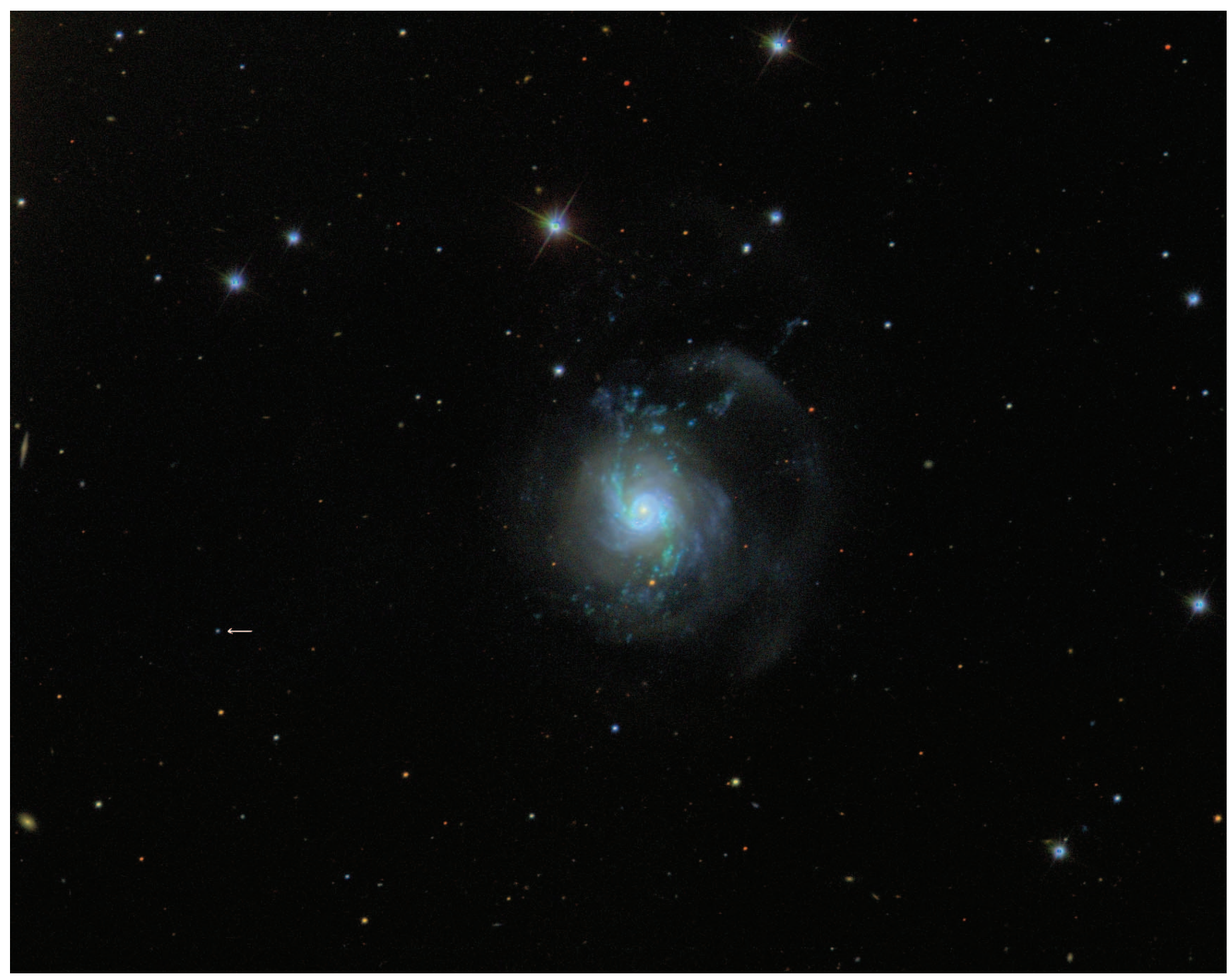

FIG. 3.-Color composite image of NGC 3310 and its environment from SDSS imaging. The image is a composite of the SDSS $g, r$, and $i$ images made using the color-weighting scheme described by Lupton et al. (2004) and measures 13.'6 $\times 9.2$. Roughly, north is up and east to the left; the image is tilted at a position angle of about $-20^{\circ}$. The super star cluster SDSS $1039+53$ is to the left of the galaxy and is indicated by an arrow. The faint red streak running down the left side of the image is due to a fifth-magnitude star to the north of the field. The color mapping is such that $\mathrm{H} \alpha$ is green and $\mathrm{H} \beta+[\mathrm{O}$ III $] 25007$ is blue, showing the presence of star-forming regions. Note the disturbed spiral arms and the inner ring of intense star formation.

$30 \mathrm{Myr}$, and de Grijs et al. suggest a burst duration of 40 Myr. Thus, SDSS $1039+53$ is considerably older than the most recent starburst event in the galaxy disk.

NGC 3310 is surrounded by shells and tidal debris (see Fig. 3), and recent observations have found additional features extending to distances of about $15 \mathrm{kpc}$ from the galaxy. Kregel \& Sancisi (2001) observed H I tails to the north and south of the galaxy, while Wehner \& Gallagher (2005) find a faint stellar arc to the north and east. These observations suggest an age for the encounter that produced the faint stellar arc of about $0.5 \mathrm{Gyr}$, close to the age inferred for SDSS $1039+53$. The position of SDSS $1039+53$ lies close to that of the faint outer stellar arc, near feature "N" in Figures 2 and 4 of Wehner \& Gallagher (2005). (This feature has a surface brightness of $\mu_{V}=22.55 \mathrm{mag} \mathrm{arcsec}^{-2}$ and is not visible in the shallower SDSS images.) Wehner \& Gallagher (2005) suggest that the outer stellar arc is tidal debris from a disrupted dwarf companion. However, our finding of a comparatively young cluster in this location suggests that the faint light could be composed of stars formed in the merger event. Obtaining accurate colors for the faint light should help clarify this situation. Although it is not necessary to invoke formation of SDSS $1039+53$ at this location - had it formed in the center of the galaxy it would have needed an average velocity in the plane of the sky with respect to the galaxy of only $35 \mathrm{~km} \mathrm{~s}^{-1}$ to reach its present location-its properties identify it with the merger event that took place in NGC 3310 and identify it as a young globular cluster associated with and formed from the far-flung debris of the merger.

While most of the super star clusters found to date in starburst dwarfs and merging galaxies are in the inner regions of the galaxies, it is also the case that for almost none of these galaxies have the surroundings been searched. Yet star formation has been observed well outside the optical disks in a small number of galaxies. For example, Hibbard et al. (2005) identify star formation in the tidal tails of the Antennae (NGC 4038/4039) in Galaxy Evolution Explorer images. Small $\mathrm{H}$ II regions have been discovered at distances as large as $30 \mathrm{kpc}$ from their galaxy (Ryan-Weber et al. 2004), and in most of the cases discussed in 

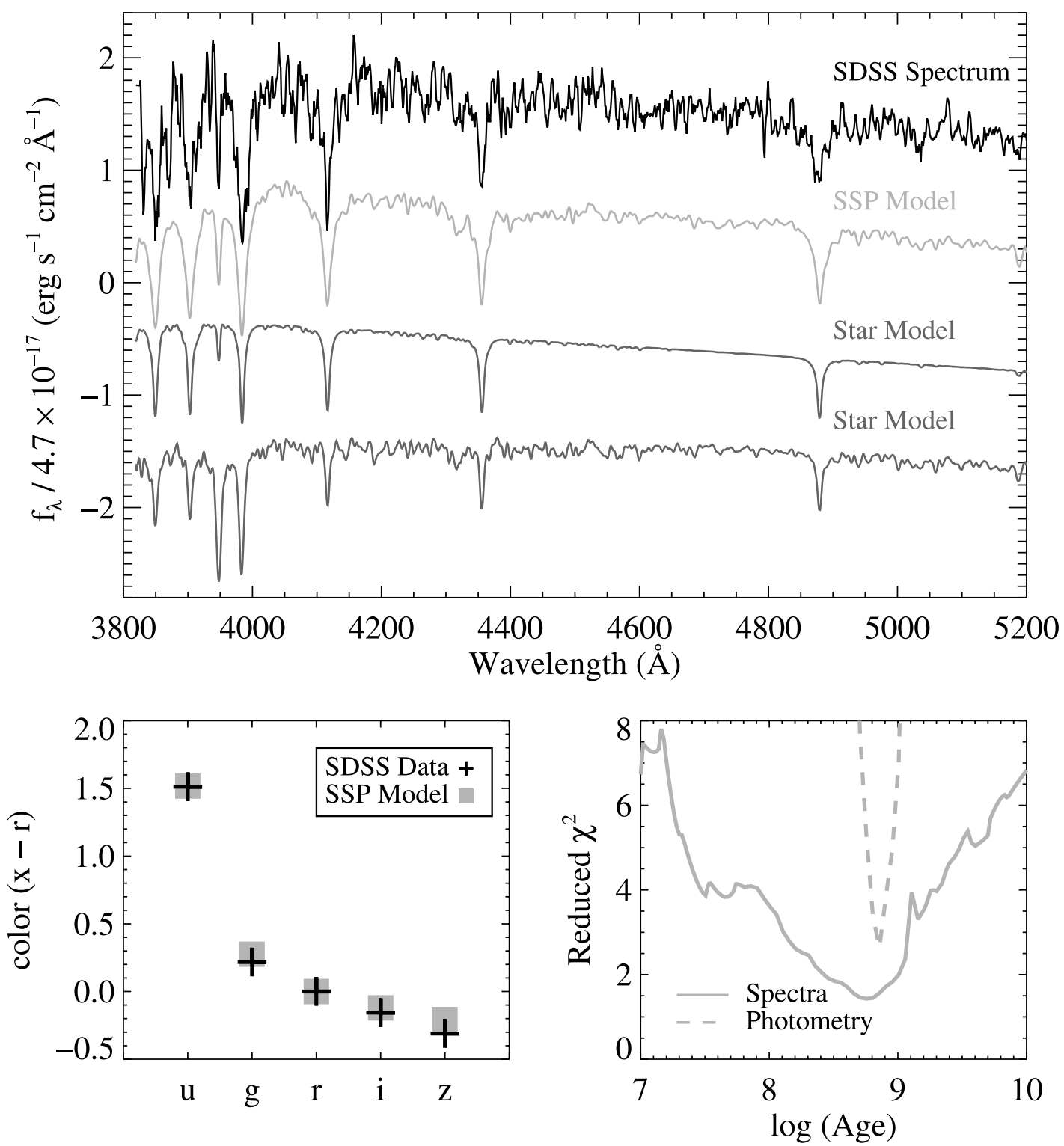

Fig. 4.- Model fits to SDSS $1039+53$. The top panel contrasts the SDSS spectrum with the best-fitting SSP model and two stellar models. The SSP model is from Bruzual \& Charlot (2003) and has an age of $570 \mathrm{Myr}$ and $Z=1 / 4 Z_{\odot}$. The stellar models are from Kurucz (1993) and have $T_{\text {eff }}=6500 \mathrm{~K}$, [Fe/H] $=-2.5$, and $\log g=2.25$ (upper) and $T_{\text {eff }}=6250 \mathrm{~K},[\mathrm{Fe} / \mathrm{H}]=-0.5$, and $\log g=-2.0$ (lower). Both the data and the models have been boxcar-smoothed by 5 pixels. The SSP model provides a considerably better fit to the Ca K line at 3934 A. The bottom left panel compares the SDSS photometry with the best-fitting SSP model. The error bars on the colors of SDSS $1039+53$ are smaller than the data points. The bottom right panel shows the evolution of the reduced $\chi^{2}$ of the fits to both the SSP spectrum (solid line) and the SSP photometric data (dashed line). The two independent fits provide some feel for the systematic errors. We estimate the cluster age to be $700 \pm 150$ Myr.

that paper there is evidence for tidal disturbance of the associated galaxy; HST imaging shows star clusters and dwarf galaxies in the tidal tails of merger galaxies that are themselves undergoing intense star formation (Knierman et al. 2003; Tran et al. 2003; Bastian et al. 2005). Thus, it is possible that there are many more super star clusters at large distances from NGC 3310 - the field (Fig. 3) contains several more sources of bluish colorand that young globular cluster systems can be identified around this and other galaxies.

Finally, we compare SDSS 1039+53 with SDSS 090745.0+ 024507, the hypervelocity star discovered by Brown et al. (2005). As Figure 2 shows, SDSS $0907+02$ is much bluer than SDSS $1039+53$, with the colors of a young star. Furthermore, there are no plausible host galaxies within many degrees. Thus, unlike SDSS 1039+53, the observational data for SDSS 0907+02 strongly support the interpretation of Brown et al. (2005): that it is a star escaping the Galaxy. So far, SDSS spectroscopy has not revealed any more hypervelocity Galactic stars.

We thank the referee for a prompt and helpful report. Partial support for the computer systems required to process and store the data was provided by NASA via grant NAG5-6734 and by Princeton University. We also thank Princeton University and the National Science Foundation (NSF) via grant AST 03-07582 for generous support. T. C. B. acknowledges partial support of this work from grants AST 04-06784 and PHY 02-16783, Physics Frontier Centers/JINA (Joint Institute for Nuclear Astrophysics), awarded by the NSF. This research made use of the IDL Astronomy User's Library at Goddard Space Flight Center. 
Funding for the creation and distribution of the SDSS Archive has been provided by the Alfred P. Sloan Foundation, the Participating Institutions, NASA, the NSF, the US Department of Energy, the Japanese Monbukagakusho, and the Max Planck Society. The SDSS Web site is http://www.sdss.org. The SDSS is managed by the Astrophysical Research Consortium for the Participating Institutions. The Participating Institutions are the University of Chicago, Fermilab, the Institute for Advanced Study, the Japan Participation Group, The Johns Hopkins University, the Korean Scientist Group, Los Alamos National Laboratory, the Max Planck Institute for Astronomy, the Max Planck Institute for Astrophysics, New Mexico State University, the University of Pittsburgh, the University of Portsmouth,
Princeton University, the United States Naval Observatory, and the University of Washington.

The Hobby-Eberly Telescope (HET) is a joint project of the University of Texas at Austin, Pennsylvania State University, Stanford University, Ludwig-Maximillians-Universität München, and Georg-August-Universität Göttingen. The HET is named in honor of its principal benefactors, William P. Hobby and Robert E. Eberly. The Marcario Low-Resolution Spectrograph is named for Mike Marcario of High Lonesome Optics, who fabricated several optics for the instrument but died before its completion; it is a joint project of the HET partnership and the Instituto de Astronomía de la Universidad Nacional Autónoma de México.
Abazajian, K., et al. 2003, AJ, 126, 2081

2004, AJ, 128, 502

2005, AJ, 129, 1755

Adelman-McCarthy, J. K., et al. 2006, ApJS, 162, 38

Allende Prieto, C., Beers, T. C., Wilhelm, R., Newberg, H. J., Rockosi, C. M.,

Yanny, B., \& Lee, Y. S. 2006, ApJ, 636, 804

Arp, H. A., \& Sandage, A. 1985, AJ, 90, 1163

Bastian, N., Hempel, M., Kissler-Patig, M., Homeier, N. L., \& Trancho, G. 2005, A\&A, 435, 65

Beers, T. C., Rossi, S., Norris, J. E., Ryan, S. G., \& Shefler, T. 1999, AJ, 117, 981

Billett, O. H., Hunter, D. A., \& Elmegreen, B. G. 2002, AJ, 123, 1454

Blanton, M. R., Lin, H., Lupton, R. H., Maley, F. M., Young, N., Zehavi, I., \& Loveday, J. 2003, AJ, 125, 2276

Brown, W. R., Geller, M. J., Kenyon, S. J., \& Kurtz, M. J. 2005, ApJ, 622, L33

Bruzual, A. G., \& Charlot, S. 2003, MNRAS, 344, 1000

Chabrier, G. 2003, PASP, 115, 763

de Grijs, R., Anders, P., Bastian, N., Lynds, R., Lamers, H. J. G. L. M., \& O’Neil, E. J. 2003a, MNRAS, 343, 1285

de Grijs, R., Fritze-von Alvensleben, U., Anders, P., Gallagher, J. S., Bastian, N., Taylor, V. A., \& Windhorst, R. 2003b, MNRAS, 342, 259

de Vaucouleurs, G., de Vaucouleurs, A., Corwin, H. G., Jr., Buta, R. J., Paturel, G., \& Fouqué, P. 1991, Third Reference Catalogue of Bright Galaxies (Berlin: Springer)

Djorgovski, S. G. 1993, in ASP Conf. Ser. 50, Structure and Dynamics of Globular Clusters, ed. S. G. Djorgovski \& G. Meylan (San Francisco: ASP), 373

Drinkwater, M. J., Gregg, M. D., Hilker, M., Bekki, K., Couch, W. J., Ferguson,

H. C., Jones, J. B., \& Phillips, S. 2003, Nature, 423, 519

Finlator, K., et al. 2000, AJ, 120, 2615

Fukugita, M., Ichikawa, T., Gunn, J. E., Doi, M., Shimasaku, K., \& Schneider, D. P. 1996 , AJ, 111,1748

Gallagher, J. S., III, \& Smith, L. J. 2004, in ASP Conf. Ser. 322, The Formation and Evolution of Massive Young Star Clusters, ed. H. G. J. L. M. Lamers, L. J. Smith, \& A. Nota (San Francisco: ASP), 149

Gallagher, S. C., Charlton, J. C., Hunsberger, S. D., Zaritsky, D., \& Whitmore, B. C. 2001, AJ, 122, 163

Gelatt, A. E., Hunter, D. A., \& Gallagher, J. S., III. 2001, PASP, 113, 142

Gunn, J. E., et al. 1998, AJ, 116, 3040 2005, AJ, submitted

Harris, W. E. 1996, AJ, 112, 1487

Hașegan, M., et al. 2005, ApJ, 627, 203

Hibbard, J. L., et al. 2005, ApJ, 619, L87

Hill, G. J., Nicklas, H. E., MacQueen, P. J., Mitsch, W., Wellem, W., Altmann,

W., Wesley, G. L., \& Ray, F. B. 1998, Proc. SPIE, 3355, 433

Ho, L. C., \& Filippenko, A. V. 1996a, ApJ, 466, L83 1996b, ApJ, 472, 600

Hogg, D. W., Finkbeiner, D. P., Schlegel, D. J., \& Gunn, J. E. 2001, AJ, 122, 2129

Holtzmann, J. A., et al. 1992, AJ, 103, 691

Ivezić, Ž., et al. 2004, Astron. Nachr., 325, 583

Knierman, K. A., Gallagher, S. C., Charlton, J. C., Hunsberger, S. D., Whitmore, B., Kundu, A., Hibbard, J. E., \& Zaritsky, D. 2003, AJ, 126, 1227

Kregel, M., \& Sancisi, R. 2001, A\&A, 376, 59

Kurucz, R. L. 1979, ApJS, 40, 1

1993, Kurucz CD-ROM 13, ATLAS9 Stellar Atmosphere Programs and $2 \mathrm{~km} / \mathrm{s}$ grid (Cambridge: $\mathrm{SAO}$ )

Lamers, H. G. J. L. M., Smith, L. J., \& Nota, A., eds. 2004, ASP Conf. Ser. 322, The Formation and Evolution of Massive Young Star Clusters (San Francisco: ASP)

Lançon, A., \& Boily, C. M., eds. 2000, ASP Conf. Ser. 211, Massive Star Clusters (San Francisco: ASP)

\section{EFERENCES}

Lipscy, S. J., \& Plavchan, P. 2004, ApJ, 603, 82

Lupton, R. H., Blanton, M. R., Fekete, G., Hogg, D. W., O’Mullane, W., Szalay, A. S., \& Wherry, N. 2004, PASP, 116, 133

Lupton, R. H., Gunn, J. E., Ivezić, Ž., Knapp, G. R., Kent, S. M., \& Yasuda, N. 2001, in ASP Conf. Proc. 238, Astronomical Data Analysis Software and Systems X, ed. F. R. Harnden, F. A. Primini, \& H. E. Payne (San Francisco: ASP), 269

Lupton, R. H., Ivezić, Ž., Gunn, J. E., Knapp, G. R., Strauss, M. A., \& Yasuda, N. 2002, Proc. SPIE, 4836, 350

McCrady, N., Graham, J. R., \& Vacca, W. D. 2005, ApJ, 621, 278

Melnick, J., Moles, M., \& Terlevich, R. 1985, A\&A, 149, L24

Melo, V. P., Muñoz-Tuñón, C., Maíz-Apellániz, J., \& Tenorio-Tagle, G. 2005, ApJ, 619, 270

Meurer, C. R., Heckman, T. M., Leitherer, C., Kinney, A., Robert, C., \& Garnett, D. R. 1995, AJ, 110, 2665

Moultaka, J., Iloaisky, S. A., Prugniel, P., \& Soubiran, C. 2004, PASP, 116, 693

Mulder, P. S., \& van Driel, W. 1996, A\&A, 309, 403

Munn, J. A., et al. 2004, AJ, 127, 3034

Newberg, H. J., et al. 2002, ApJ, 569, 245

O'Connell, R. W. 2004, in ASP Conf. Ser. 322, The Formation and Evolution of Massive Young Star Clusters, ed. H. G. J. L. M. Lamers, L. J. Smith, \& A. Nota (San Francisco: ASP), 551

O’Connell, R. W., Gallagher, J. S., \& Hunter, D. A. 1994, ApJ, 433, 65

Ojha, D. K. 2001, MNRAS, 322, 426

Oke, J. B., \& Gunn, J. E. 1983, ApJ, 266, 713

Pastoriza, M. G., Dottori, H. A., Terlevich, E., Terlevich, R., \& Diaz, A. I. 1993, MNRAS, 260, 177

Peterson, C. J. 1993, in ASP Conf. Ser. 50, Structure and Dynamics of Globular Clusters, ed. S. G. Djorgovski \& G. Meylan (San Francisco: ASP), 337

Pier, J. R., Munn, J. A., Hindsley, R. B., Hennessy, G. S., Kent, S. M., Lupton, R. H., \& Ivezić, Ž. 2003, AJ, 125, 1559

Prugniel, P., \& Soubiran, C. 2001, A\&A, 369, 1048

Ryan-Weber, E. V., et al. 2004, AJ, 127, 1431

Sánchez-Portal, M., Díaz, Á. I., Terlevich, E., \& Terlevich, R. 2004, MNRAS, 350,1087

Schlegel, D. J., Finkbeiner, D. P., \& Davis, M. 1998, ApJ, 500, 525

Schweizer, F. 1986, Science, 231, 227

Sirko, E., et al. 2004a, AJ, 127, 899 2004b, AJ, 127, 914

Smith, J. A., et al. 2002, AJ, 123, 2121

SubbaRao, M., Frieman, J. A., Bernardi, M., Loveday, J., Nichol, R. C., Castander, F., \& Meiksin, A. 2002, Proc. SPIE, 4847, 452

Tran, H. D., et al. 2003, ApJ, 585, 750

Tremonti, C. A., Calzetti, D., Leitherer, C., \& Heckman, T. 2001, ApJ, 555, 322

Tucker, D. L., et al. 2005, PASP, submitted

Turner, J. L., \& Beck, S. C. 2004, ApJ, 602, L85

Uomoto, A., et al. 1999, BAAS, 31, 1501

van den Bergh, S. 1971, A\&A, 12, 474

Vanzi, L. 2003, A\&A, 408, 523

Wehner, E. H., \& Gallagher, J. S., III. 2005, ApJ, 618, L21

Whitmore, B. C., \& Schweizer, F. 1995, AJ, 109, 960

Whitmore, B. C., Schweizer, F., Leitherer, C., Borne, K., \& Robert, C. 1993, AJ, 106, 1354

Wilhelm, R., Beers, T. C., \& Gray, R. O. 1999, AJ, 117, 2308

Yanny, B., et al. 2000, ApJ, 540, 825

York, D. G., et al. 2000, AJ, 120, 1579

Zepf, S. E., Ashman, K. M., English, J., Freeman, K. C., \& Sharples, R. M. 1999, AJ, 118, 752 